\title{
Simultaneous occurrence of systemic lupus erythematosus and coeliac disease-like features
}

\author{
Yakov Varkel ${ }^{1}$, Andrei Braester ${ }^{1}$, Harry Suprun ${ }^{2}$, David Nusem ${ }^{1}$ and Yvona Horn ${ }^{1}$ \\ 'Department of Medicine 'A' and ${ }^{2}$ Department of Pathology and Cytology, Western Galilee Regional Hospital, \\ Naharyia, Israel
}

\begin{abstract}
Summary: Involvement of the gastrointestinal tract in systemic lupus erythematosus is well documented but small intestinal malabsorption is a rare event. We report a 27 year old woman with systemic lupus erythematosus who had malabsorption and coeliac-like changes on intestinal biopsy. This is the eighth reported case of this association the significance of which is discussed.
\end{abstract}

\section{Introduction}

Small bowel malabsorption in systemic lupus erythematosus (SLE) with coeliac-like features on jejunal biopsy has received little attention and only seven patients with this association have been described previously. We report a further case and discuss the association.

\section{Case report}

A 27 year old Arab woman was admitted to the Department of Internal Medicine because of anaemia and a facial rash. Six months earlier she had developed microcytic, hypochromic anaemia when $\mathrm{X}$-ray studies of the gastrointestinal tract were normal. There was no subsequent response to prolonged oral iron. Two months before admission she developed intermittent episodes of arthralgia of the wrists with morning stiffness. On admission the temperature was $37^{\circ} \mathrm{C}$, blood pressure $120 / 80 \mathrm{mmHg}$ but there were no other findings apart from a butterfly facial rash and a systolic murmur $2 / 6$ audible over the left sternal border. There was no weight loss or steatorrhoea clinically.

Investigations: haemoglobin $6.6 \mathrm{~g} / \mathrm{dl}$, white cell count $3.4 \times 10^{9} / 1$, platelets $300 \times 10^{9} / 1$, erythrocyte sedimentation rate (ESR) $80 \mathrm{~mm} / \mathrm{h}$. The blood urea nitrogen (BUN), electrolytes, creatinine, glucose, albumin, globulin, liver enzymes, alkaline phosphatase, phosphorus were in the normal range. The serum calcium was $1.9 \mathrm{mmol} / 1$ (normal $2.1-2.6 \mathrm{mmol} / \mathrm{l}$ ). The

Correspondence: A. Braester, M.D., Rh. Zalman Shneur 13, Ramot Remez, 32543 Haifa, Israel

Accepted: 3 March 1989 serum iron was $5 \mu \mathrm{mol} / \mathrm{l}$ (normal 9-26.9 $\mu \mathrm{mol} / \mathrm{l}$ ). Proteinuria was $0.6 \mathrm{~g} / 24 \mathrm{~h}$ and the creatinine clearance $37 \mathrm{ml} / \mathrm{min}$ (normal $70-120 \mathrm{ml} / \mathrm{min}$ ). The serum folic acid was borderline low at $5.9 \mathrm{nmol} / \mathrm{l}$ with a raised total iron binding capacity of $68 \mu \mathrm{mol} / 1$ (normal 49-61 $\mu \mathrm{mol} / \mathrm{l})$. Immunological studies revealed an antinuclear antibody titre of $1: 100$, negative antidouble stranded DNA, and Coombs' tests low levels of the third $(0.3 \mathrm{~g} / \mathrm{l}$, normal $0.8-1.8 \mathrm{~g} / \mathrm{l})$ and fourth $(0.19 \mathrm{~g} / \mathrm{l}$, normal $0.20-0.45 \mathrm{~g} / \mathrm{l})$ components of complement, negative serological tests for syphilis and for rheumatoid factor. The serum electrophoresis revealed polyclonal increase of gammaglobulin. Bone marrow aspiration showed no stainable iron and an oral iron loading test showed malabsorption of iron. D-Xylose tolerance test showed urinary excretion of $21.1 \mathrm{mmol} / 5 \mathrm{hours}$ (normal $33-53 \mathrm{mmol}$ ). Total fat excretion after 3 days on a diet of $100 \mathrm{~g}$ of fat daily was $7.4 \mathrm{~g} /$ day (normal less than $5 \mathrm{~g} /$ day). The Schilling test showed normal absorption of vitamin $B_{12}$. Jejunal biopsy showed features consistent with grade I gluteninduced enteropathy (Figure 1).

In spite of the suspicion of SLE, but without enough criteria to arrive at such diagnosis, we concluded that the anaemia was due to coeliac disease. The patient was discharged and placed on a gluten-free diet. Two months later there was no improvement in her malabsorption tests and the haemoglobin was only $6.8 \mathrm{~g} / \mathrm{dl}$. This time the titre of antibody to negative double stranded DNA was 1:80. Renal biopsy showed diffuse moderate proliferative glomerulonephritis. A diagnosis of SLE, therefore, was made and the gluten-free diet was stopped. The patient was treated with cyclophosphamide and prednisone with good clinical improvement. A repeat small bowel biopsy 3 months

(C) The Fellowship of Postgraduate Medicine, 1989 


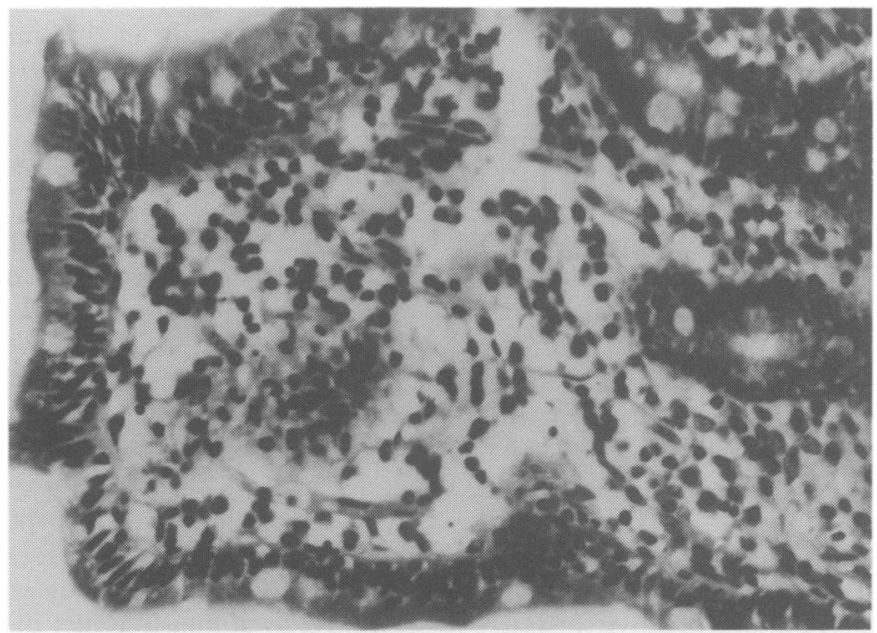

Figure 1 Jejunal biopsy before the treatment. Slight blunting of villi $(H \& E \times 50)$.

later showed normal jejunal villi (Figure 2). The haemoglobin 6 months later was $12 \mathrm{~g} / \mathrm{dl}$ and the serum calcium concentration normal.

\section{Discussion}

Involvement of the gastrointestinal tract in SLE is well documented. The disorders described include serositis, enteritis, pancreatitis, haemorrhage, ulcerative colitis, oesophageal diseases, malabsorption and protein losing enteropathy, and liver disease. ${ }^{1}$ It is, however, unclear if gastrointestinal involvement is a consistent feature of SLE, as is nephritis or cerebritis, or if it represents a sporadic problem.

The occurrence of morphological alterations in the small intestine with malabsorption appears to be rare. A Medline search of English language publications has revealed only seven well documented cases. ${ }^{2-6}$ The small bowel biopsy showed villous abnormalities, subtotal $\left({ }^{2-5}\right.$ and the present case) or total villous atrophy. ${ }^{2,6}$

There are several theories for the occurrence of malabsorption in SLE. One is that the malabsorption state is coincidental. However, in the series of Siurala ${ }^{2}$ there was a high incidence $(10.7 \%)$ of SLE patients

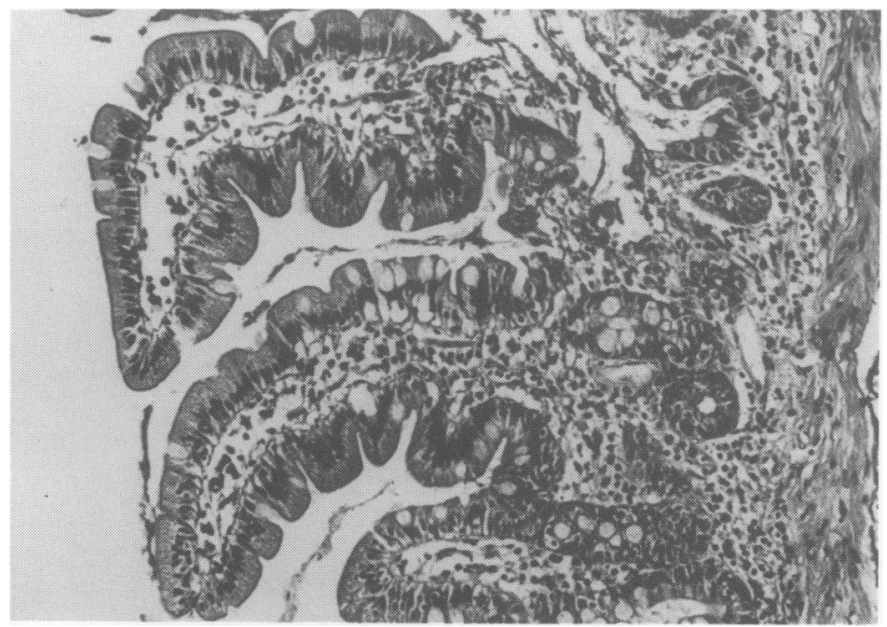

Figure 2 Jejunal biopsy after treatment. Normal jejunal villi, lamina propria and contents $(\mathrm{H} \& \mathrm{E} \times 25)$. 
with malabsorption which suggests the disorders are not recurring together by chance. Siurala describes seven patients with collagen diseases and concomitant malabsorption with small intestine changes of coeliac disease, three of whom had SLE. Three patients, including one with SLE, were treated with gluten-free diet. The SLE patient did not respond, the two other patients had symptomatic and objective improvement. In two patients ( ${ }^{5}$ and the present case) a gluten-free diet was also tried, but without improvement occurring.

In one case, ${ }^{4}$ coeliac-like changes on intestinal biopsy occurred in a patient with SLE and dermatitis herpetiformis (DH), with normal small bowel function. The biopsy was not repeated on a gluten-free diet.

In another case, ${ }^{6}$ a patient with SLE developed coeliac disease with clinical and histological improvement after treatment with gluten-free diet. Histocompatibility antigen (HLA) typing was positive for HLA B8 and DR3 (which are both present in a greater percentage of patients with SLE and with coeliac disease than a normal population). HLA typing has not been done in other published cases.

Weisman ${ }^{5}$ reported a patient with small intestinal

\section{References}

1. Hoffman, B.I. \& Katz, W.A. The gastrointestinal manifestation of systemic lupus erythematosus: a review of the literature. Arthritis Rheum 1980, 9: 237-247.

2. Siurala, M., Julkunen, H., Toivonen, S. et al. Digestive tract in collagen diseases. Acta Med Scand 1965, 178: 13-25.

3. Bazinet, P. \& Marin, G.A. Malabsorption in systemic lupus erythematosus. Am J Dig Dis 1971, 16: 460-466.

4. Davies, M.G. \& Marks, R. Simultaneous systemic lupus erythematosus and dermatitis herpetiformis. Arch Dermatol 1976, 112: 1292-1294.

5. Weisman, M.H., McDonald, E.C. \& Wilson, C.B. Studies of the pathogenesis of interstitial cystitis, obstructive uropathy and intestinal malabsorption in a patient with systemic lupus erythematosus. Am J Med 1981, 70: 875-881. malabsorption and SLE, with evidence of immune complex deposition in the blood vessel walls of the jejunum. The malabsorption state responded dramatically to steroid treatment, with reversal of the histological changes. The author suggested that immunecomplex deposition may have been responsible for the intestinal injury.

In the case of Bazinet ${ }^{3}$ vascular damage of the small bowel, stasis and bacterial overgrowth were considered responsible.

Another possible aetiology is ischaemic mucosal damage as mesenteric insufficiency from atherosclerosis may produce malabsorption. ${ }^{7}$ It has been noted that as ischaemic mucosa recovers, blunting or loss of villi are seen on biopsy. ${ }^{8}$

In conclusion, malabsorption with coeliac-like features on intestinal biopsy associated with SLE is not always due to coeliac disease, as there is a lack of response to gluten-free diet in the majority of cases.

It is also possible that a more careful search for malabsorption in patients with SLE, especially in those with gastrointestinal manifestations, might reveal a higher incidence than that reported to date.

6. Rustgi, A.K. \& Peppercorn, M.A. Gluten-sensitive enteropathy and systemic lupus erythematosus. Arch Int Med 1988, 148: 1583-1584.

7. Dardik, H., Seidenberg, B., Parker, J.G. et al. Intestinal angina with malabsorption treated by elective revascularization. JAMA 1965, 194: 148-152.

8. Spiro, H.M. Clinical Gastroenterology, 2nd edition. Macmillan Publishing, New York, 1977, p. 460. 